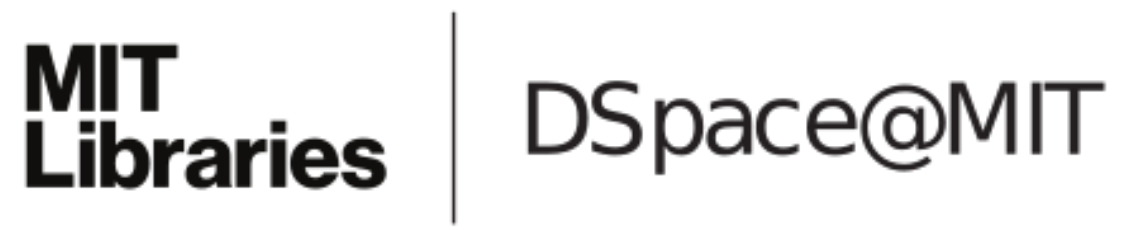

\author{
MIT Open Access Articles
}

The role of scaffold microarchitecture in engineering endothelial cell immunomodulation

The MIT Faculty has made this article openly available. Please share how this access benefits you. Your story matters.

Citation: Indolfi, Laura, Aaron B. Baker, and Elazer R. Edelman. "The Role of Scaffold Microarchitecture in Engineering Endothelial Cell Immunomodulation." Biomaterials 33, no. 29 (October 2012): 7019-7027.

As Published: http://dx.doi.org/10.1016/j.biomaterials.2012.06.052

Publisher: Elsevier

Persistent URL: http://hdl.handle.net/1721.1/102308

Version: Author's final manuscript: final author's manuscript post peer review, without publisher's formatting or copy editing

Terms of use: Creative Commons Attribution-Noncommercial-NoDerivatives 


\title{
The role of scaffold microarchitecture in engineering endothelial cell immunomodulation
}

\author{
Laura Indolfi ${ }^{\mathrm{a},{ }^{*}}$, Aaron B. Baker ${ }^{\mathrm{b}}$, and Elazer R. Edelman ${ }^{\mathrm{a}, \mathrm{c}}$ \\ Laura Indolfi: lindolfi@mit.edu \\ aHarvard-MIT Division of Health Science and Technology, Massachusetts Institute of Technology, \\ Cambridge, MA, USA \\ bDepartment of Biomedical Engineering, Cockrell School of Engineering, University of Texas at \\ Austin, Austin, TX, USA \\ ${ }^{\circ}$ Cardiovascular Division, Department of Medicine, Brigham and Women's Hospital, Harvard \\ Medical School, Boston, MA, USA
}

\begin{abstract}
The implantation of matrix-embedded endothelial cells (MEECs) has been reported to have great therapeutic potential in controlling the vascular response to injury and maintaining patency in arteriovenous anastomoses. While there is an appreciation of their effectiveness in clinical and animal studies, the mechanisms through which they mediate these powerful effects remain relatively unknown. In this work, we examined the hypothesis that the 3-dimensional microarchitecture of the tissue engineering scaffold was a key regulator of endothelial behavior in MEEC constructs. Notably, we found that ECs in porous collagen scaffold had a markedly altered cytoskeletal structure with oriented actin fibers and rearrangement of the focal adhesion proteins in comparison to cells grown on 2D surfaces. We examined the immunomodulatory capabilities of MEECs and discovered that they were able to reduce the recruitment of monocytes to an inflamed endothelial monolayer by 5 -fold compared to EC on 2D surfaces. An analysis of secreted factors from the cells revealed an 8-fold lower release of Monocyte Chemotactic Protein-1 (MCP-1) from MEECs. Differences between 3D and 2D cultured cells were abolished in the presence of inhibitors to the focal adhesion associated signaling molecule Src suggesting that adhesionmediated signaling is essential in controlling the potent immunomodulatory effects of MEEC.
\end{abstract}

\section{Keywords}

Endothelial cells morphology; Inflammation; Gelatin scaffold; Cell signaling; Surface topography; Microstructure

\section{Introduction}

Endothelial cells (ECs) are powerful mediators of many aspects of arterial biology including vascular tone, thrombosis and inflammation [1]. The loss of endothelial integrity and function drives the pathophysiological mechanisms leading to atherosclerosis, instent

(C) 2012 Elsevier Ltd. All rights reserved.

*Corresponding author. Tel.: +1 6177152060.

Authorship contributions and disclosure of conflicts of interest: L. Indolfi conceived the ideas, designed the research, performed experiments, analyzed data and wrote the paper. A.B. Baker and E.R. Edelman designed research, analyzed data and wrote the paper. The authors report no conflicts. 
restenosis and stroke [2]. As a consequence, it is an appealing strategy to implant or inject healthy endothelial cells in a diseased or injured region to modulate vascular remodeling and homeostasis. Previous studies have shown that xenogenic and allogenic matrix-embedded ECs (MEECs) can be perivascularly delivered to tailor the response to injury and the remodeling of arteriovenous anastomoses [3-6]. A fascinating aspect of MEECs is that these cells produce enhanced levels of soluble factors that regulate both the local arterial homeostasis and immunobiology [7] and even xenogeneic ECs do not induce a significant host immune response [8]. Yet, the specific role of scaffold properties in driving these changes in cell functions and in-vivo outcomes remained unclear.

Both within arteries and in tissue-engineered constructs, the dynamic interactions between ECs and the surrounding environment are potent modulator of endothelial function. The specific extracellular matrix is linked to the cell interior and contractile cytoskeleton through focal adhesion complexes [9] and changes in the local features detected by the cells can be associated with modulation of signaling pathways via mechanosensors [10]. Stiffness $[11,12]$ and chemical composition $[13,14]$ of the underlying surface of culture are among the key factors able to drive cellular behavior. Recent studies have added evidence that the 3D microarchitecture of scaffolds is itself a potent mediator of functions for cell with a surfaceadherent phenotype $[15,16]$. Therefore, in this study we examined the role of 3D microarchitecture of the tissue-engineered scaffold in regulating the immunomodulatory secretions of MEECs.

In-vivo the endothelium is the cellular gatekeeper of the tissue, being a physical barrier and a regulator of inflammatory cell entry in tissues. Monocytes are recruited from the blood flow by activated endothelial cells through a complex, highly regulated process involving both soluble factors and cell adhesion receptors [17]. Monocyte Chemotactic Protein-1 (MCP-1) is a key cytokine in immune cell recruitment by endothelial cells and a major factor in determining monocyte adhesion to the inflamed endothelium [18]. After recruitment, these cells differentiate into macrophages that are key players in the immune activation that drives atherosclerosis and restenosis [19].

In this study, we examined the relationship between scaffold architecture, cytoskeletal rearrangement, cell signaling and the production of inflammatory-related chemokines by endothelial cells grown on gelatin sponges.

\section{Materials and methods}

\subsection{Materials}

All materials were purchased from Sigma Corp. unless otherwise specified. The Src inhibitor (4-amino-5-(4-chlorophenyl)-7-(dimethylethyl)pyrazolo [3,4-d] pyrimidine) (PP2) was purchased from EMD Chemicals.

\subsection{Cell culture}

Human aortic endothelial cells (ECs) and human umbilical vein endothelial cells (HUVECs) pooled from 3 donors were grown in endothelial growth medium supplemented with EGM-2 growth supplements (Lonza). Human monocytic THP-1 cell line (American Type Culture Collection) were grown in RPMI-1640 medium with $10 \%$ FBS and $0.05 \mathrm{mM} 2-$ mercaptoethanol. ECs were grown on gelatin-coated tissue culture plates (2D-ECs) $(0.1 \%$ gelatin type A, Sigma, St Louis, MO) or in 3D gelatin matrices (MEECs) (Gelfoam, Pfizer, New York, NY). For cell-matrix engraftment, compressed sponges were cut into $1 \times 1 \times 0.3$ $\mathrm{cm}$ blocks and hydrated in culture medium at $37^{\circ} \mathrm{C}$ for $\geq 4 \mathrm{~h}$. Then $4.5 \times 10^{4} \mathrm{ECs}$ (suspended in $\sim 50 \mu \mathrm{L}$ media) were seeded onto one surface of the hydrated matrix, allowed $1.5 \mathrm{~h}$ to attach before turning the matrix over and seeding an additional $4.5 \times 10^{4}$ in growth 
media. After a further $1.5 \mathrm{~h}$ of incubation each piece was added to a separate $30 \mathrm{~mL}$ polypropylene tube containing $10 \mathrm{~mL}$ of culture medium. Matrices were cultured for up to 3 weeks, with media changed every $48-72 \mathrm{~h}$, under standard culture conditions $\left(37^{\circ} \mathrm{C}\right.$ humidified environment with $5 \% \mathrm{CO}_{2}$ ).

\subsection{Characterization of tissue engineering scaffold microarchitecture}

Three-dimensional matrices of denaturated collagen (Gelfoam, Pfizer, New York, NY) were used as 3D scaffold for EC culture. Morphological characterization of the scaffolds was carried out using an environmental Scanning Electron Microscope (eSEM; Philips/FEI XL30 FEG-SEM). Matrices were visualized in their hydrated state using low vacuum settings to preserve the architecture of the scaffolds. Porosity of the scaffold was established through serial cryosectioning of the matrix in $40 \mu \mathrm{m}$ slices and subsequent staining with Biebrich's Scarlet Acid Fuchsin dye (IMEB). Images were taken using a nikon epifluorescence microscope (inverted Eclipse Ti-E, Nikon) and processed using ImageJ software to determine the maximum diameter of the pores.

\subsection{Cell morphology and immunostaining}

EC morphology in both 2D and 3D environments was visualized by eSEM. Briefly, cells were fixed in $4 \%$ paraformaldehyde overnight and counterstained for $30 \mathrm{~min}$ with $0.5 \%$ uranyl acetate solution to increase visibility under microscope. Samples were then analyzed using a back scatter mode in low vacuum environment. For immunofluorescence analysis, cells were fixed in $4 \%$ paraformaldehyde, EC-engrafted matrices were additionally incubated in $30 \%$ sucrose, frozen and cryosectioned in $40 \mu \mathrm{m}$ slides. The cytoskeleton was visualized by staining cells for filamentous actin using fluorescent-phalloidin (Sigma). Cells were exposed to $0.2 \mathrm{M}$ glycine for $10 \mathrm{~min}$ and incubated with $0.2 \%$ triton X-100 in phosphate buffered saline for $10 \mathrm{~min}$. Goat serum (4\%) in phosphate buffered saline with $1 \%$ bovine serum albumin was applied for $1 \mathrm{~h}$ at room temperature (RT). Vinculin primary antibody (1:50, Santa Cruz, Santa Cruz, CA) was applied to the cells overnight at $4{ }^{\circ} \mathrm{C}$. Secondary antibody, alexa fluoro 488 (1:50, Invitrogen, Carlsbad, CA), was applied to the cells for $1 \mathrm{~h}$ at RT with or without rhodamine-phalloidin (1:250, Invitrogen) for visualization of F-actin. Cells were mounted with VectaShield containing DAPI (Vector Labs, Burlingame, CA). Imaging was performed via confocal microscopy (Zeiss LSM510, Germany, Confocal Core Facility at the Beth Israel Deaconess Medical Center, Harvard Medical School, Boston, MA). In the case of ECs within 3D matrices, multiple z-stack imagining was carried out due to cells lying on different focal planes (Movie S1); threedimensional rendering of the raw data was then performed using the confocal image analysis software (Movie S2). Quantification of vinculin inside the cell was carried out by fluorescence intensity analysis. Single cell area was selected and the intensity levels of the green channel were detected using the confocal image analysis software. This process was repeated for each condition of cell culture (standard and Src-inhibited 2D and 3D settings, $n$ $=20)$.

Supplementary video related to this article can be found online at http://dx.doi. org/10.1016/ j.biomaterials.2012.06.052.

\subsection{Quantitative morphology}

Images of fluorescently labeled ECs, both in 2D and 3D settings, were analyzed to determine the orientation of actin filaments. Individual cells were selected and the value of the angle $\theta$, defined as the angle between each actin filaments and the major axis of the cell, was evaluated. To normalize the results we chose to represent the data in term of cosine of $\theta$, spanning between 0 (filaments orthogonal to the axis direction) and 1 (filaments parallel to the axis direction). To have additional quantitative analysis on cytoskeleton remodeling, 
the lattice defined by the actin filaments was determined highlighting the directions achieved by the filaments together with the number of nodes, i.e. point of connection between two filaments, in each cell. It is worth to notice that analysis of ECs within matrices was performed with the help of the 3D rendering of the $z$-stack data to reduce misjudgments due to planar projection of the different focal planes.

\subsection{Biosecretion analysis}

Conditioned media of confluent 2D-ECs (at 85-90\% confluence) or MEECs (in culture for 14 days) were collected and analyzed for Monocyte Chemotactic Protein-1 (MCP-1) chemokine level by commercially available ELISA kit according to the manufacturers' instructions. To obtain the baseline/background level of soluble factor in the medium, fresh EGM-2 medium was incubated for $24 \mathrm{~h}$ without cells and measured in parallel with medium exposed to cells. This baseline/background level in the medium without cells was subtracted from the levels in medium exposed to cells to obtain the actual levels of soluble factors produced by the cells alone.

\subsection{Monocyte adhesion assay}

HUVECs were maintained on 24-well plates in EGM-2 medium and stimulated with $10 \mathrm{ng} /$ $\mathrm{mL}$ tumor necrosis factor-a (TNF-a) (Sigma) for $4 \mathrm{~h}$, then switched to control EGM-2 media. Thirty minutes prior assay, THP-1 cells in culture were washed and resuspended in serum free media before being labeled with a solution of Calcein-AM $(7.5 \mathrm{mM})$ and incubated at $37^{\circ} \mathrm{C}$, in the dark for $30 \mathrm{~min}$. Labeled cells were then washed three times in fresh RPMI-1640 serum free medium at $5.10^{6}$ cells $/ \mathrm{mL}$, and $100 \mu \mathrm{L}$ of the cell suspension $\left(5.10^{5}\right.$ cells) was loaded on TNF-a-treated HUVECs. After $1 \mathrm{~h}$ of culture, non-adherent THP- 1 cells were removed by washing three times with RPMI-1640 medium. Adherent cells were lysed for $10 \mathrm{~min}$ in $0.1 \%$ SDS in PBS and lysates transferred to optically neutral 96 well plates. The fluorescence of adherent THP-1 cells was measured with a fluorescence microplate reader. To investigate the role that morphology-induced secreted factors play on inflammatory response, adhesion of fluorescently labeled THP-1 cells on activated HUVEC monolayers was quantified in the presence of standard planar ECs or MEECs conditioned media. Two sets of experiments were performed and their layout is reported for clarity (Fig. 1). In the first setting, HUVEC monolayer was exposed to conditioned media gathered from $2 \mathrm{D}$ or 3D-cultured ECs simultaneously to the treatment with TNF- $a$ prior to perform the adhesion test. In a parallel experiment the monocytes were pre-incubated with the conditioned media from standard ECs or MEECs for 30 min after labeling with Calcein$\mathrm{AM}$, washed in serum free media to remove excess of conditioned media and put in contact with the TNF-a stimulated HUVECs monolayer, then the protocol followed as described above.

\subsection{Cell signaling inhibition}

Morphological analysis and cytoskeletal organization were assessed for ECs cultured on flat or contoured domains in the presence for $24 \mathrm{~h}$ of a $10 \mathrm{mM}$ final concentration solution of Src inhibitor PP2. All the above described set of experiments were also performed using Src-inhibited-ECs, in those cases the media containing PP2 was changed with fresh media for additional $24 \mathrm{~h}$ to avoid direct interaction of the inhibitor.

\subsection{Statistical analysis}

All results are shown as mean \pm SEM. Multiple comparisons between groups were analyzed by 2 -way ANOVA followed by a Tukey post-hoc test. Differences were considered significant at $p<0.05$. 


\section{Results}

\subsection{Scaffold microarchitecture and EC cytoskeletal structure}

We used a foamed gelatin scaffold for 3D ECs culture as this material has been used in both animal and clinical trials of MEECs [3,20,21]. We first determined the microarchitecture of the material using quantitative morphology followed by eSEM analysis. Analysis of pore dimension carried out from cryosectioned slides showed a wide distribution with a peak in the range $50-150 \mu \mathrm{m}$ (Fig. 2). It is notable that the single struts of the scaffold have dimensions ranging from 10 to $50 \mu \mathrm{m}$ (Fig. 3A-B), on the same order of magnitude as the cell itself, and cells seeded into the scaffolds conformed closely to the underlying microarchitecture (Fig. 3C-D and Movie S2). In contrast, ECs on 2D substrata had a cobblestone appearance (Fig. 3E-F).

The size correspondence between cell and matrix strut diameter induces ECs to achieve themselves a 3D morphology (3D-MEECs), while keeping the same cell area than in 2D settings (data not shown), and determines changes in actin fiber organization (Fig. 3G); both changes depend strongly on local substratum patterning. ECs circumferentially wrap around matrix struts with dimension smaller than themselves, showing actin filaments oriented perpendicular to the long direction of the strut (Fig. 3Hi) and parallel to the direction of bending. As strut dimensions rise and exceed the dimensions of the ECs, cells align their actin filaments increasingly parallel to the longest aspect of the strut (Fig. 3Hii). Actin fibers are therefore always oriented parallel to the major direction of curvature (Fig. 4A) and never haphazardly arranged as shown by a picked distribution of $\cos \theta$ (Fig. 4B) thus determining one major filament direction and two nodes at the polar extremes of the cells consistent with a "gripping" nature of ECs on textured 3D surfaces (Fig. 4C). In contrast, ECs in 2D culture "spread" and therefore manifest the dense peripheral actin organization defined in standard cell culture on planar surface (Fig. 3I). Filaments in flat 2D-ECs circumscribe the periphery of the cell avoiding crossing the center with a broader distribution of fiber orientation (Fig. $4 \mathrm{~A}-\mathrm{B})$ and the presence of diamond-like lattice defined by 4 filament directions and 4 nodes (Fig. 4C).

We also examined the distribution of focal adhesion complexes in ECs under different culture environments by immunofluorescent staining for vinculin. Vinculin focal adhesion protein in flat 2D-ECs is arranged in a punctate organization located at the edge of the actin filaments (Fig. 5A-i), a co-localization consistent with vinculin's role as anchorage to planar culture substrata [22]. In contrast, such co-localization is not present in contoured 3DMEECs, where the vinculin is distributed predominantly perinuclearly (Fig. 5A-ii) as highlighted by the differences in fluorescence intensity associated with the protein. In fact, when vinculin is organized in plaques, as in the case of 2D-ECs, the fluorescent signals is almost 3-fold lower than when it is perinuclearly distributed in 3D-MEECs (Fig. 5B).

\subsection{Correlation between microarchitecture and cell signaling}

To test our hypothesis that specific architecture of the substratum can interfere with cell signaling, we treated cells with Src kinase inhibitor (PP2) for $24 \mathrm{~h}$ and quantified the alterations in cytoskeletal rearrangement for both 2D and 3D culture. Visual differences in morphology were confirmed with a quantitative analysis of fiber orientation, as bundle filaments were completely oriented along the direction of the major axis of the Src-inhibited flat 2D-ECs (Fig. 4A-B). These cells lost the characteristic diamond-like lattice and attained the dual node configuration (Fig. 4C). Intriguingly, the pattern of intracellular vinculin changed from punctate lesions at filament edges to a more perinuclear organization with higher fluorescent signal (Fig. 5B). Src-inhibited 2D-EC became in all senses much like fiber alignment and vinculin distribution attained in contoured 3D-MEECs without Src 
inhibition (Fig. 5A-i and A-iii). Src inhibition, however, had no significant effect on the organization and intensity of vinculin or alignment of actin in 3D-MEECs (Figs. 4 and 5A$i v$ ), perhaps as additional effect cannot be induced above and beyond that already imposed by the substratum.

\subsection{Regulation of monocyte adhesion through soluble factors}

Local immune suppression by MEECs is thought to be a major mechanism in the effectiveness of these constructs in inhibiting restenosis and maintaining graft patency $[5,6,23]$. In typical application MEECs are implanted perivascularly to the target tissue; as a consequence the soluble factors produced by the MEECs are critical in the efficacy of such cell therapy. We collected conditioned media (CM) from ECs cultured in scaffold and on 2D substrates and then used it to incubate TNF- $a$ stimulated endothelial monolayers. Both 2Dand 3D-cultured ECs decreased the adherence of monocytes to the activated monolayer (Fig. 6A), with CM from 3D-MEECs being about 5-fold more inhibitory. When we used CM gathered from ECs in Src-inhibited 2D and 3D settings, this increased the anti-adherent properties of the CM by $20 \%$ for $2 \mathrm{D}$ culture while no statistical difference was found for $3 \mathrm{D}$ culture (Fig. 6A). This result was consistent with our hypothesis that 3D microarchitecture triggers Src inactivation and that reduction in Src kinase signaling leads to a biosecretion that is more suppressive to monocyte adherence.

To examine whether the $\mathrm{CM}$ could also have a direct effect on the inflammatory cells, we used it to treat the monocytes and examined their adherence to TNF-a stimulated endothelial monolayer. In this case there was an even stronger outcome on monocyte adherence control with a 6-fold lower adhesion for 3D-MEECs compared to standard 2D culture. Src inhibition on the 2D-ECs and 3D-MEECs also induced the CM to a greater antiadhesive direct effect on monocytes (Fig. 6B). Intriguingly, this effect leads the inhibited 2D-EC to similar levels of adhesion than standard, uninhibited 3D-MEEC. Moreover, CM of Src-inhibited 3D-MEECs promote an additional effect when in direct contact with inflammatory cells abolishing almost completely monocyte adhesion. These results together demonstrate that the soluble factors from 3D-MEECs have a powerful immunosuppressive effect both on the vascular monolayer and the circulating monocytes themselves.

\subsection{Analysis of MCP-1 secretion}

EC secretion of MCP-1 is an important factor regulating monocyte adhesion to inflamed vascular monolayers [18]. Intrigued by our results on control of monocyte adhesion by 3DMEECs, we investigated the secretion of MCP-1 to determine the cause of such important outcome. Fascinatingly, ECs in 3D matrices release 8-fold less MCP-1 than in 2D culture (Fig. 6C). After $24 \mathrm{~h}$ incubation with the Src inhibitor PP2, biosecretion was notably suppressed for both cell culture settings. Most significantly, Src inhibition induced 2D-ECs to reduce MCP-1 secretion exactly to the same level observed with non-treated 3D-MEECs (Fig. 6C, $p=$ N.S.). Incubation with fresh media for additional $24 \mathrm{~h}$ did not affect these levels, indicating a sustained phenotype change.

\section{Discussion}

At its most basic level the cardiovascular system is composed of an intricate equilibrium of cell-cell and cell-substratum interactions. Imbalance in vascular cell autocrine and paracrine regulation elicits a cascade of events including thrombosis, aberrant proliferation and dysregulation of the inflammatory system. Matrix-embedded endothelial cells (MEECs) are an emerging therapy for reducing the effects of the inflammatory response to vascular injury $[6,24]$ and maintaining patency in arteriovenous grafts [3,21]. In addition, xenografted endothelial cells implanted in a matrix scaffold elicit little host immune response [25]. 
While these studies demonstrate strong immunomodulatory effects, it is still unclear what mechanism elicits immune suppression and how these activities depend on the properties of the embedding scaffold. This study sought to examine the relation between scaffold architecture and the effectiveness of MEECs in inhibiting immune cell recruitment.

Cells seeded within 3D scaffolds sense substratum dimensions and contour on a microarchitectural scale and achieve cell morphologies from relatively flat to far more complex arrangements. The majority of the available tissue engineering scaffolds are characterized by a surface of contact whose dimensions are situated at the extremes of a size range relative to the cell. Consequently, the morphology of the cell itself in these constructs tends to be planar due to the culture topology either being very large compared to the cells size (e.g. hydrogel) or very small (e.g. nano-fiber meshes) and is thus perceived as a continuum by the cells $[26,27]$. On the contrary, the gelatin scaffolds used in our study have topological features on the order of the cell size and consequently facilitate a threedimensional cellular morphology unseen before that includes bending and deformation during attachment, thus eliciting strong alterations in cytoskeletal organization. Flat 2D-ECs exhibited the characteristic cobblestone morphology with a dense peripheral actin distribution of the filaments [22] while contoured MEECs in 3D sponges have a remodeled cytoskeleton, with almost all the bundles parallel to a fixed direction. This change in morphology is peculiar and in net contrast with results shown in other studies where EC are grown on different substratum architecture [26,28]. Intriguingly, this ordered configuration of the actin cytoskeleton is more similar to that induced in healthy endothelial monolayer in in-vivo settings [29].

Endothelial cell anchorage to the specific extracellular environment is mediated through the interactions of integrins and focal adhesion complexes (FAC) incorporating proteins such as vinculin [30]. In our studies, staining for the FAC-associated protein vinculin demonstrated that there were increased amounts of focal adhesions in the cells with a 3D morphology. Cells reside in a variety of different natural environments, fully embedded within extracellular matrix or with only one surface in contact with the subjacent environment. Fraley et al. [31] showed that loss of actin-vinculin co-localization occurs when cells are fully embedded within 3D hydrogel scaffolds. We now demonstrate that this is the case also for cells with a defined apical and basal surface like ECs, where 3D contoured conformational morphology, dictated by substratum-induced mechanical remodeling of the cytoskeleton, drives a perinuclear vinculin distribution. Together these findings support that matrix embedding in a scaffold with topological features close to the cell size causes increased focal adhesion and cytoskeletal remodeling to accommodate the cell bending to align with scaffold topology.

A key hypothesis for the in-vivo effects of MEECs lies in their potent immunomodulatory activities. Since focal adhesions are responsible for strong cell-substratum adhesion and transmit information in a bidirectional manner between extracellular molecules and cell cytoplasm [32] and as we had seen significant alterations in adhesion-mediated mechanisms with culture of cells in our 3D matrices, we sought to link these effects to the immunomodulatory factors produced by MEECs. To do this we first harvested the soluble factors produced by 3D-MEECs and 2D-ECs and applied them to confluent endothelial monolayer that had been stimulated with TNF-a. We then examined the adhesion of cultured monocytes to the treated monolayers. This study founds a 5 -fold decrease in monocyte adhesion to MEECs-treated cell monolayers, demonstrating the strong immunosuppressive activity of 3D-MEEC soluble factors. In a similar experiment we treated the monocytes instead of the endothelial monolayers with MEEC-derived factors and found a similar trend in monocyte adhesion. Together these results suggest that MEECs can 
alter the immune response both by affecting the vessel endothelium and circulating inflammatory cells.

MCP-1 is a potent soluble factor that is essential to promote and regulate the migration of monocytes [18], and in this work we explored the potential of substratum-induced morphological changes in 3D-MEECs to be responsible of inducing paracrine control inflammation through regulation of this cytokine. Our studies indeed demonstrated that 3DMEECs produced 8-fold less MCP-1 than 2D cultures and this production could be further reduced in both groups by treatment with a Src-inhibitor. Src kinase family, known to play a role in signaling transduction of endothelial barrier dysfunction [33] as well as being involved in inflammatory-related pathways [34], has been implicated in regulating FACmediated signaling and cytoskeletal rearrangement [35]. The protein Src is associated with focal adhesion activation, vinculin interactions [36] and involved in pathways controlling inflammation [37]. In this study we demonstrated that substratum architecture affects cell signaling by interfering with the Src pathway through alteration in focal adhesion protein organization. Most significantly, Src inhibition induced 2D-ECs not only to similar morphology but also to secrete the same level of MCP-1 as non-treated 3D-MEECs and in turn caused a reduction in monocyte adhesion.

Together these findings are consistent with a Src-mediated pathway that is regulated by local topology and leads to alterations in MCP-1 thereby hindering monocyte adhesion (Fig. 7).

\section{Conclusion}

Our work elucidates the mechanisms through which the interaction with a contoured topology alters the EC biosecretory profile and explores whether such differences are linked to inhibition/activation of intracellular signaling. We demonstrate that scaffold microtopology is a potent regulator of MEEC function and can indeed tune the immunosuppressive properties of these cells. Our study further reveals that MEECs can act through paracrine mechanisms to modulate the biology of both monocyte and the activated endothelium separately. These paracrine factors are strongly regulated through a Srcdependent signaling pathway that can be altered by the matrix scaffold itself. These insights may provide a means to further optimize the properties of MEEC-based therapies by allowing the rational design of scaffold morphology to produce greater immunosuppressive effects. It is our hope that further delineation of EC-matrix architecture interactions will propel the design of cell-therapies for vascular, chronic inflammatory, cancer and autoimmune diseases whose morbidity and mortality is prevalent worldwide.

\section{Supplementary Material}

Refer to Web version on PubMed Central for supplementary material.

\section{Acknowledgments}

The authors thank Matthew C. Canver for his help in analyzing actin fiber orientation. This work was supported in part by a grant from the National Institutes of Health (to Dr. E. Edelman, R01 GM 49039) and by the American Heart Association (to Dr. A. Baker, Scientist Development Grant 10SDG2630139).

\section{References}

1. Ribatti D, Nico B, Vacca A, Roncali L, Dammacco F. Endothelial cell heterogeneity and organ specificity. J Hematother Stem Cell Res. 2002; 11(1):81-90. [PubMed: 11847005]

2. Michiels C. Endothelial cell functions. J Cell Physiol. 2003; 196(3):430-43. [PubMed: 12891700] 
3. Nugent HM, Sjin RT, White D, Milton LG, Manson RJ, Lawson JH, et al. Adventitial endothelial implants reduce matrix metalloproteinase-2 expression and increase luminal diameter in porcine arteriovenous grafts. J Vasc Surg. 2007; 46(3):548-56. [PubMed: 17826244]

4. Nugent HM, Ng YS, White D, Groothius A, Kanner G, Edelman ER. Delivery site of perivascular endothelial cell matrices determines control of stenosis in a porcine femoral stent model. J Vasc Interv Radiol. 2009; 20(12):1617-24. [PubMed: 19854069]

5. Nugent HM, Rogers C, Edelman ER. Endothelial implants inhibit intimal hyperplasia after porcine angioplasty. Circ Res. 1999; 84(4):384-91. [PubMed: 10066672]

6. Nugent HM, Edelman ER. Endothelial implants provide long-term control of vascular repair in a porcine model of arterial injury. J Surg Res. 2001; 99(2):228-34. [PubMed: 11469891]

7. Methe H, Hess S, Edelman ER. Endothelial immunogenicity-a matter of matrix microarchitecture. Thromb Haemost. 2007; 98(2):278-82. [PubMed: 17721607]

8. Methe H, Groothuis A, Sayegh MH, Edelman ER. Matrix adherence of endothelial cells attenuates immune reactivity: induction of hyporesponsiveness in allo- and xenogeneic models. Faseb J. 2007; 21(7):1515-26. [PubMed: 17264166]

9. Sinha RK, Tuan RS. Regulation of human osteoblast integrin expression by orthopedic implant materials. Bone. 1996; 18(5):451-7. [PubMed: 8739903]

10. Biggs MJ, Richards RG, Wilkinson CD, Dalby MJ. Focal adhesion interactions with topographical structures: a novel method for immuno-SEM labelling of focal adhesions in S-phase cells. J Microsc. 2008; 231(Pt 1):28-37. [PubMed: 18638187]

11. Gilchrist CL, Darling EM, Chen J, Setton LA. Extracellular matrix ligand and stiffness modulate immature nucleus pulposus cell-cell interactions. PLoS One. 2011; 6(11):e27170. [PubMed: 22087260]

12. Kocgozlu L, Rabineau M, Koenig G, Haikel Y, Schaaf P, Freund JN, et al. The control of chromosome segregation during mitosis in epithelial cells by substrate elasticity. Biomaterials. 2012; 33(3):798-809. [PubMed: 22041225]

13. Kolodziej CM, Kim SH, Broyer RM, Saxer SS, Decker CG, Maynard HD. Combination of integrin-binding peptide and growth factor promotes cell adhesion on electron-beam-fabricated patterns. J Am Chem Soc. 2012; 134(1):247-55. [PubMed: 22126191]

14. Chew SY, Low WC. Scaffold-based approach to direct stem cell neural and cardiovascular differentiation: an analysis of physical and biochemical effects. J Biomed Mater Res A. 2011; 97(3):355-74. [PubMed: 21448997]

15. Sero JE, Thodeti CK, Mammoto A, Bakal C, Thomas S, Ingber DE. Paxillin mediates sensing of physical cues and regulates directional cell motility by controlling lamellipodia positioning. PLoS One. 2011; 6(12):e28303. [PubMed: 22194823]

16. Geisse NA, Sheehy SP, Parker KK. Control of myocyte remodeling in vitro with engineered substrates. In Vitro Cell Dev Biol Anim. 2009; 45(7):343-50. [PubMed: 19252956]

17. Wittchen ES. Endothelial signaling in paracellular and transcellular leukocyte transmigration. Front Biosci. 2009; 14:2522-45. [PubMed: 19273217]

18. Reape TJ, Groot PH. Chemokines and atherosclerosis. Atherosclerosis. 1999; 147(2):213-25. [PubMed: 10559506]

19. Hansson GK. Inflammatory mechanisms in atherosclerosis. J Thromb Haemost. 2009; 7(Suppl. 1): 328-31. [PubMed: 19630827]

20. Conte MS, Nugent HM, Gaccione P, Guleria I, Roy-Chaudhury P, Lawson JH. Multicenter phase $\mathrm{I} / \mathrm{II}$ trial of the safety of allogeneic endothelial cell implants after the creation of arteriovenous access for hemodialysis use: the V-HEALTH study. J Vasc Surg. 2009; 50(6):1359-68. e1. [PubMed: 19958986]

21. Nugent HM, Groothuis A, Seifert P, Guerraro JL, Nedelman M, Mohanakumar T, et al. Perivascular endothelial implants inhibit intimal hyperplasia in a model of arteriovenous fistulae: a safety and efficacy study in the pig. J Vasc Res. 2002; 39(6):524-33. [PubMed: 12566978]

22. Wong MK, Gotlieb AI. Endothelial cell monolayer integrity. I. Characterization of dense peripheral band of microfilaments. Arteriosclerosis. 1986; 6(2):212-9. [PubMed: 3954675] 
23. Methe H, Nugent HM, Groothuis A, Seifert P, Sayegh MH, Edelman ER. Matrix embedding alters the immune response against endothelial cells in vitro and in vivo. Circulation. 2005; 112(9 Suppl):I89-95. [PubMed: 16159871]

24. Nathan A, Nugent MA, Edelman ER. Tissue engineered perivascular endothelial cell implants regulate vascular injury. Proc Natl Acad Sci U S A. 1995; 92(18):8130-4. [PubMed: 7667257]

25. Methe H, Hess S, Edelman ER. The effect of three-dimensional matrix-embedding of endothelial cells on the humoral and cellular immune response. Semin Immunol. 2008; 20(2):117-22. [PubMed: 18243732]

26. Santos MI, Tuzlakoglu K, Fuchs S, Gomes ME, Peters K, Unger RE, et al. Endothelial cell colonization and angiogenic potential of combined nano- and micro-fibrous scaffolds for bone tissue engineering. Biomaterials. 2008; 29(32):4306-13. [PubMed: 18706689]

27. Millon L, Padavan D, Hamilton A, Boughner D, Wan W. Exploring cell compatibility of a fibronectin-functionalized physically crosslinked poly(vinyl alcohol) hydrogel. J Biomed Mater Res B Appl Biomater. 2012; 100(1):1-11. [PubMed: 21998037]

28. Kemeny SF, Figueroa DS, Andrews AM, Barbee KA, Clyne AM. Glycated collagen alters endothelial cell actin alignment and nitric oxide release in response to fluid shear stress. $\mathrm{J}$ Biomech. 2011; 44(10):1927-35. [PubMed: 21555127]

29. White GE, Gimbrone MA Jr, Fujiwara K. Factors influencing the expression of stress fibers in vascular endothelial cells in situ. J Cell Biol. 1983; 97(2):416-24. [PubMed: 6684121]

30. Zebda N, Dubrovskyi O, Birukov KG. Focal adhesion kinase regulation of mechanotransduction and its impact on endothelial cell functions. Microvasc Res. 2012; 83(1):71-81. [PubMed: 21741394]

31. Fraley SI, Feng Y, Krishnamurthy R, Kim DH, Celedon A, Longmore GD, et al. A distinctive role for focal adhesion proteins in three-dimensional cell motility. Nat Cell Biol. 2010; 12(6):598-604. [PubMed: 20473295]

32. Schwartz MA. Integrin signaling revisited. Trends Cell Biol. 2001; 11(12):466-70. [PubMed: 11719050]

33. Tinsley JH, Ustinova EE, Xu W, Yuan SY. Src-dependent, neutrophil-mediated vascular hyperpermeability and beta-catenin modification. Am J Physiol Cell Physiol. 2002; 283(6):C1745-51. [PubMed: 12388068]

34. Cao X, Tay A, Guy GR, Tan YH. Activation and association of Stat3 with Src in v-Srctransformed cell lines. Mol Cell Biol. 1996; 16(4):1595-603. [PubMed: 8657134]

35. Zhang Z, Izaguirre G, Lin SY, Lee HY, Schaefer E, Haimovich B. The phosphorylation of vinculin on tyrosine residues 100 and 1065, mediated by SRC kinases, affects cell spreading. Mol Biol Cell. 2004; 15(9):4234-47. [PubMed: 15229287]

36. Sefton BM, Hunter T, Ball EH, Singer SJ. Vinculin: a cytoskeletal target of the transforming protein of Rous sarcoma virus. Cell. 1981; 24(1):165-74. [PubMed: 6263485]

37. Funakoshi-Tago M, Tago K, Andoh K, Sonoda Y, Tominaga S, Kasahara T. Functional role of cSrc in IL-1-induced NF-kappa B activation: c-Src is a component of the IKK complex. J Biochem. 2005; 137(2):189-97. [PubMed: 15749833] 
Basal level with EGM-2 media
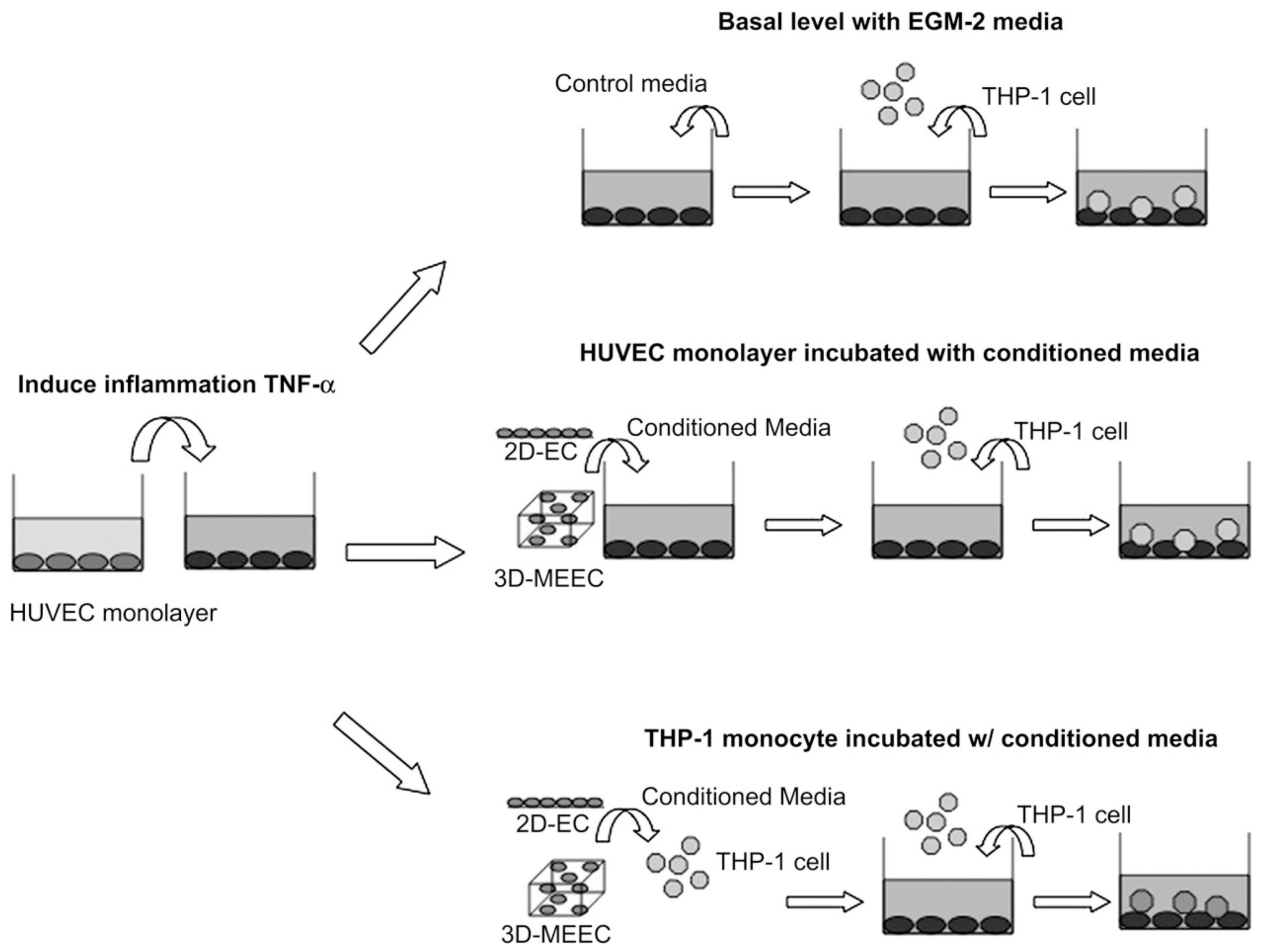

Fig. 1.

Experimental layout. A confluent endothelial monolayer (HUVECs) was activated by incubation with $10 \mathrm{ng} / \mathrm{mL}$ of TNF-a for $4 \mathrm{~h}$. Top scheme: Thereafter, basal levels of THP-1 monocyte adhesion were analyzed by switching to EGM-2 media and incubate HUVEC monolayers with fluorescently labeled monocyte for $1 \mathrm{~h}$ before reading intensity of adherent cells. Two parallel sets of experiments were performed. Middle scheme: HUVECs were preincubated with conditioned media from flat 2D-ECs or contoured 3D-MEECs, then media changed to control EGM-2 and THP-1 added in suspension for $1 \mathrm{~h}$ before reading intensity of adherent cells. Bottom scheme: The same rational was pursued for monocytes, THP-1 cells were incubated with conditioned media from 2D-ECs or 3D-MEECs prior to use in the adhesion test. 
A

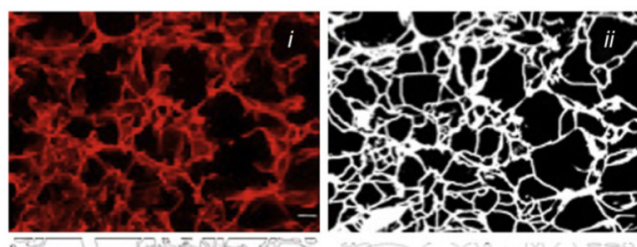

in $x$ Q

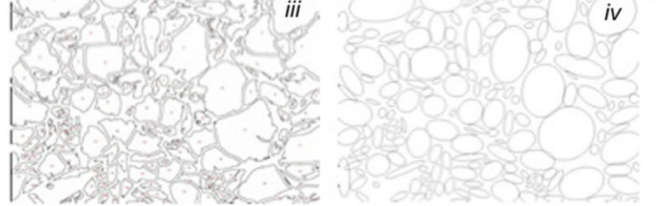

B

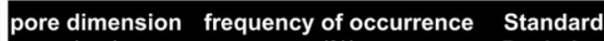

$(\mu \mathrm{m}) \quad(\%) \quad$ Deviation

$\leq 50$

$50-100$

11.49

45.60

45.60

22.22

11.51 1.27

$100-150$

1.10

$\geq 200$

Fig. 2.

Quantification of scaffold porosity. (A) Scheme of the layout used to determine scaffold pore size. 3D collagen-based scaffolds have been cryosectioned in $40 \mu \mathrm{m}$ slide, stained with red dye and images recorded using a fluorescent microscope to determine the porosity of the mesh (i). Fluorescent micrographs were converted in binary images (ii), where each black area represented a pore (iii). Finally pore shape was approximated to an ellipsoid (iv) and the length of the major axis was evaluated. (B) Porosity analysis of the 3D collagen-based scaffold. Dimensions were grouped in range of $50 \mu \mathrm{m}$ each ranging from 50 to $200 \mu \mathrm{m}$. The frequency of occurrence and the standard deviation of the analysis were then evaluated. 

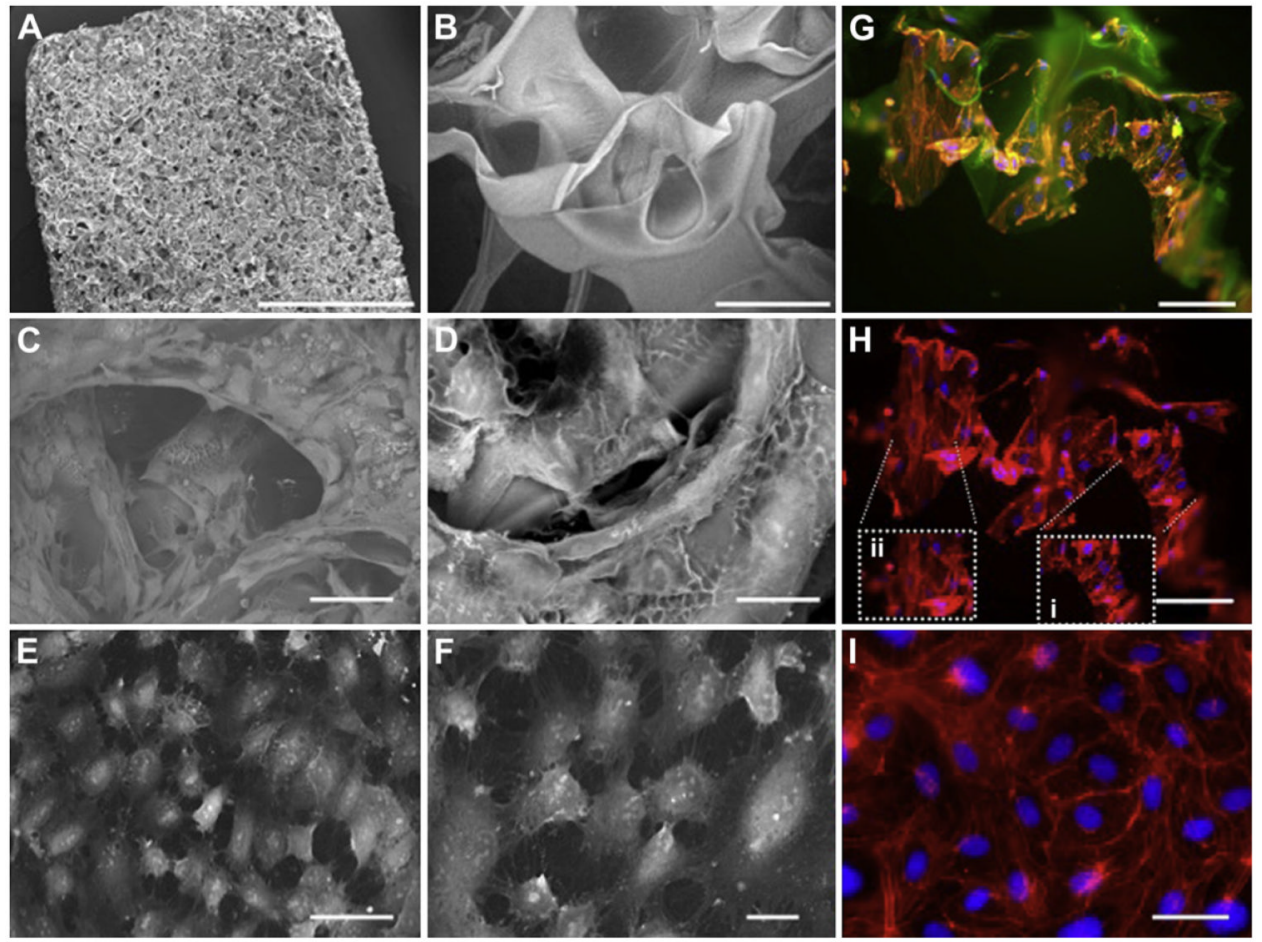

Fig. 3.

Microarchitecture of the substratum determines morphology and cytoskeleton rearrangement of ECs seeded in 2D and 3D domains. (A) Environmental-SEM micrographs of gelatin 3D scaffolds. (B) An individual struts at higher magnification. (C-D) 3D matrices seeded with ECs after 14 days induce a peculiar three-dimensional morphology of cells. (E-F) ECs seeded on planar 2D substrata attain their well-described cobblestone flat morphology. (G) Immunofluorescent images of ECs seeded on 3D matrixes better highlight the 3D contoured arrangement of cells induced by the underlying substratum: actin filaments (red), nuclei (blue) and gelatin matrix (green autofluorescence). (H) Cytoskeletal rearrangement is determined by the specific dimension of the cell substratum inducing the cell to wrap (insert i) or to bend (insert ii) around matrix struts. (I) The cytoskeleton of ECs on 2D is characterized by a diamond-like organization of the actin filaments. Scale bar: $3 \mathrm{~mm}(\mathrm{~A})$; $100 \mu \mathrm{m}$ (B); $50 \mu \mathrm{m}$ (C,E,G,H,I); and $20 \mu \mathrm{m}$ (D,F). (For interpretation of the references to color in this figure legend, the reader is referred to the web version of this article.) 


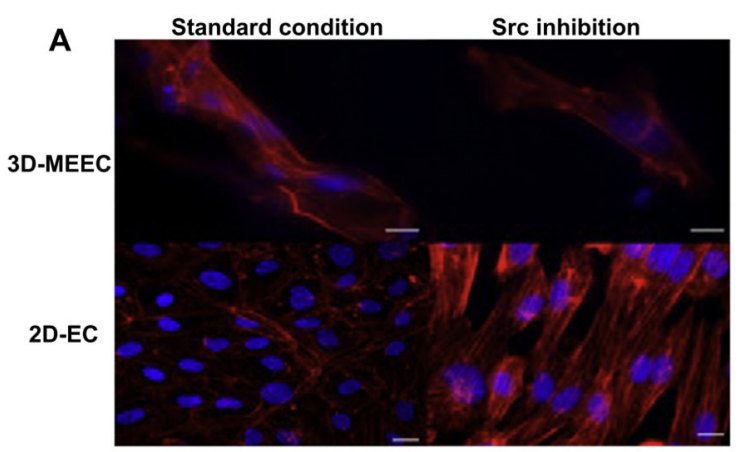

\section{C}

2D-EC

Diamond-like configuration 4 nodes (A, B, C, D) 4 directions of filaments $(\mathrm{AB}, \mathrm{BC}, \mathrm{CD}, \mathrm{DA})$

3D-MEEC, Src-inhibited EC (2D and 3D)

Polarized configuration 2 nodes (A, B)

1 direction of filaments $(A B)$

\section{B}

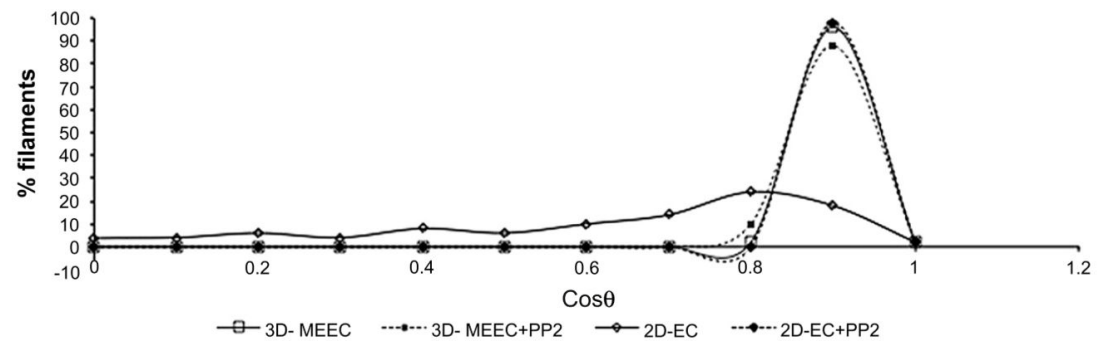

Fig. 4.

Actin filaments orientation strongly depends on the substratum of culture and it is related to inhibition of Src pathway. (A) Fluorescent images of actin orientation for ECs cultured on $2 \mathrm{D}$ or $3 \mathrm{D}$ substrata both in standard condition and after inhibition of Src pathway (Scale bar is $10 \mu \mathrm{m}$ for 3D-MEECs, and $20 \mu \mathrm{m}$ for 2D-ECs). It is worth to notice that analysis of ECs within matrices was performed with the help of the 3D rendering of the $z$-stack data to reduce misjudgments due to planar projection of the different focal planes. (B) Distribution of actin filaments orientation in terms of angle $\theta$ spanned respect to a fixed direction, e.g. major axis in the cell, for 2D-ECs and 3D-MEEC in standard and Src-inhibited conditions (C) 2D-ECs exhibit under standard condition a peripheral dense distribution of the actin filaments. Cytoskeleton defines a diamond-like lattice, with four different directions of the fibers, mainly in the peripheral edges of the cell, and four connection points, e.g. nodes (top). Conversely 3D-MEECs are characterized by a more polarized configuration, with actin filaments predominantly parallel to the major axis of the cell. In this setting, fibers are oriented only in one direction with the presence of two nodes at the extremity (bottom). Inhibition of Src pathway determines such polarized configuration of the cytoskeleton in both settings (bottom). $\theta$ is the angle spanned between the filament and the major axis of the cells as depicted in the figure. 

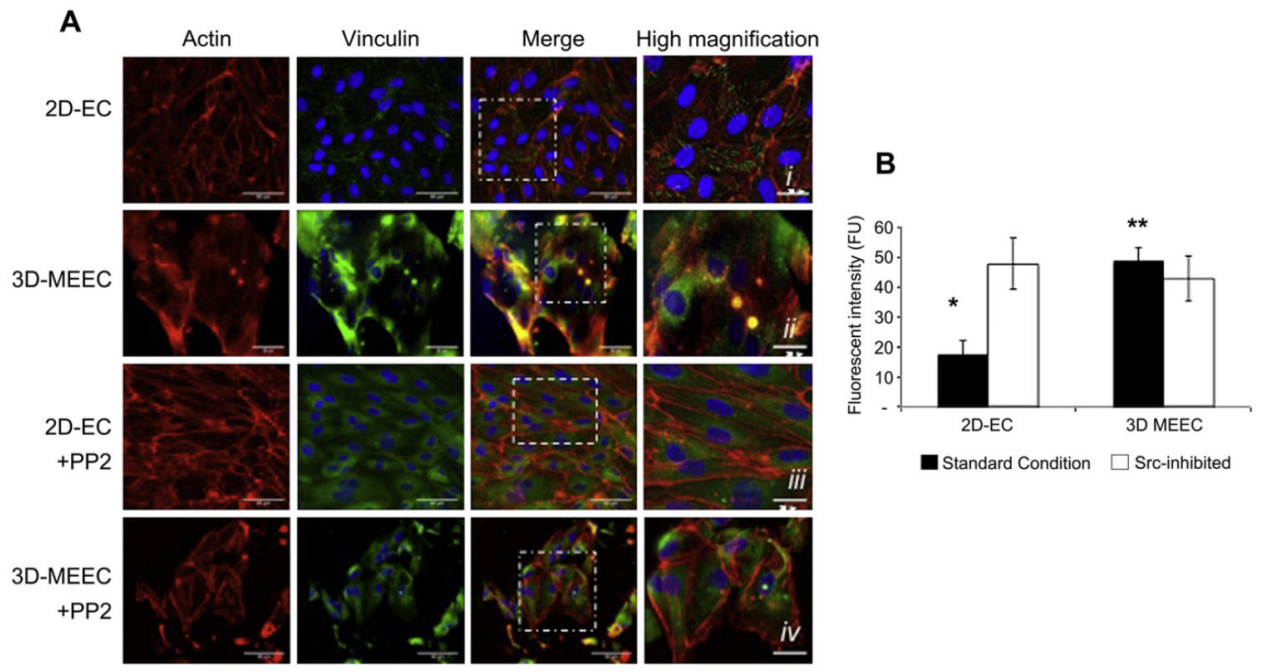

Fig. 5.

Substratum architecture of ECs culture induces changes in focal adhesion protein localization through inhibition of Src pathway. A) Immunofluorescent micrographs for ECs seeded in $2 D$ or $3 D$ settings: actin (red), vinculin (green) and nuclei (blue). Merged images show as vinculin is colocalized at the edge of actin fibers for flat 2D-ECs (i), while on contoured 3D-MEECs vinculin arrangement is perinuclear (ii). After incubation with a Src inhibitor (PP2) vinculin for 2D-ECs attains a perinuclear localization (iii) such as in the baseline 3D-MEECs (ii). Treatment of 3D-MEECs with PP2 does not significantly alter vinculin subcellular localization (iv). Scale bar: $50 \mu \mathrm{m}$. Inserts scale bar $i-i v .25 \mu \mathrm{m}$. B) Vinculin quantification through an analysis of fluorescence intensity within single cell. Area of individual cell was selected and the intensity of the signal in the green channel was determined using the confocal software. 2D-ECs showed a mean signal of vinculin fluorescence that was 3 -fold lower than the intensity from 3 D-MEECs ${ }^{*} p<0.05$, versus all other groups). In contrast, when cells were incubated with Src inhibitor PP2, the signals among the two different cultures was identical and equal to the level of standard 3D-MEECs (** $p>0.05$ versus Src-inhibited $2 \mathrm{D}$ and $3 \mathrm{D}$ settings). (For interpretation of the references to color in this figure legend, the reader is referred to the web version of this article.) 
A

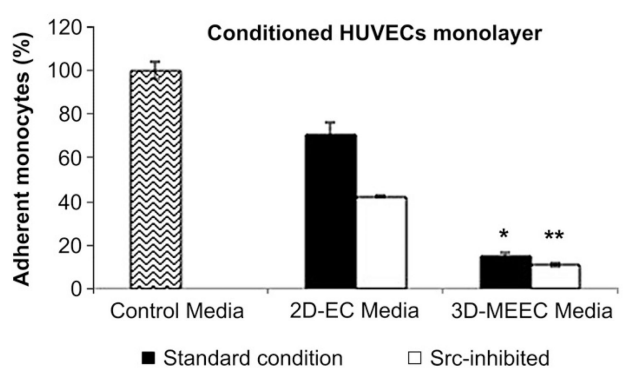

B

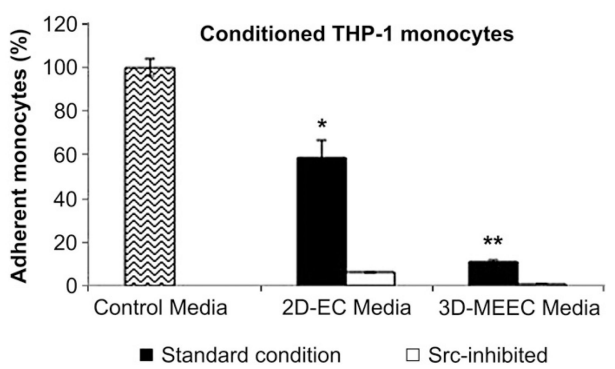

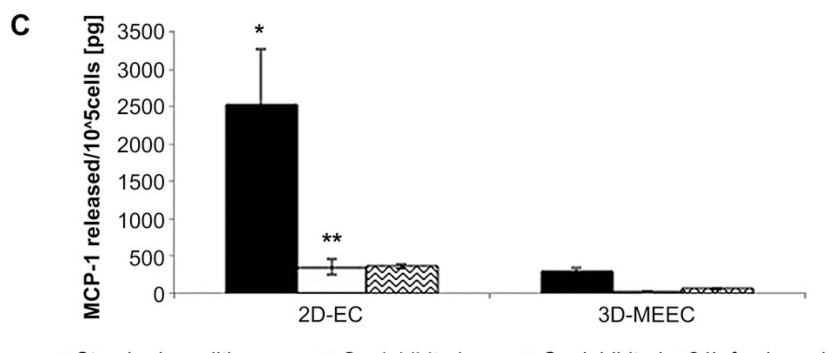

Fig. 6.

Substratum-induced ECs morphology affects biosecretory regulation of monocyte adhesion and depends upon Src inhibition. A) When flat 2D-ECs conditioned media (CM) was used to incubate vascular endothelial monolayer activated with $\mathrm{TNF}-\boldsymbol{\alpha}$ a reduction in monocytes adhesion of $30 \%$ respect to control was achieved. However, when contoured 3D-MEECs media was used, the percent inhibition of monocyte adherence increased to almost 5-fold. CM from flat 2D-ECs incubated with Src inhibitor PP2 reduced monocyte adhesion of over $50 \%$ respect to control. When Src-inhibited contoured 3D-MEECs media was used, a reduction higher than $80 \%$ was achieved $(* p$ value $<0.005$ versus all other groups; $* * p$ value $>0.05$ versus standard 3D-MEEC). B) In a parallel set of experiments, in which CM was used to incubate monocytes prior to the adhesion test, a similar trend although with a higher percentage of adhesion inhibition with respect to control was shown. Both 2D- and 3D-MEECs treated with PP2 induced $90 \%$ less monocyte adhesion that control (* $p$ value < 0.05 versus all other groups; $* * p$ value $>0.05$ versus Src-inhibited 2D-EC). C) MCP-1 secretion by ECs is modulated by microarchitecture and requires Src signaling. ECs in 3D matrices release 8 -fold less MCP-1 $\left(289 \pm 61 \mathrm{pg} / 10^{5}\right.$ cells $)$ than in $2 \mathrm{D}$ culture $(2526 \pm 740$ $\mathrm{pg} / 10^{5}$ cells). 24-h incubation with Src inhibitor PP2 drastically reduced secretion of MCP-1 either in flat 2D-ECs $\left(328 \pm 102 \mathrm{pg} / 10^{5}\right.$ cells $)$ and contoured 3D-MEECs $\left(30 \pm 3 \mathrm{pg} / 10^{5}\right.$ cells). Incubation with fresh media for additional $24 \mathrm{~h}$ did not affect these levels $(360 \pm 28$ versus $57 \pm 9 \mathrm{pg} / 10^{5}$ cells, $2 \mathrm{D}$ and $3 \mathrm{D}$ respectively). (* $p$ value $<0.05$ versus all other groups; ** $p$ value $>0.05$ versus standard $3 \mathrm{D}-\mathrm{MEEC})$. 


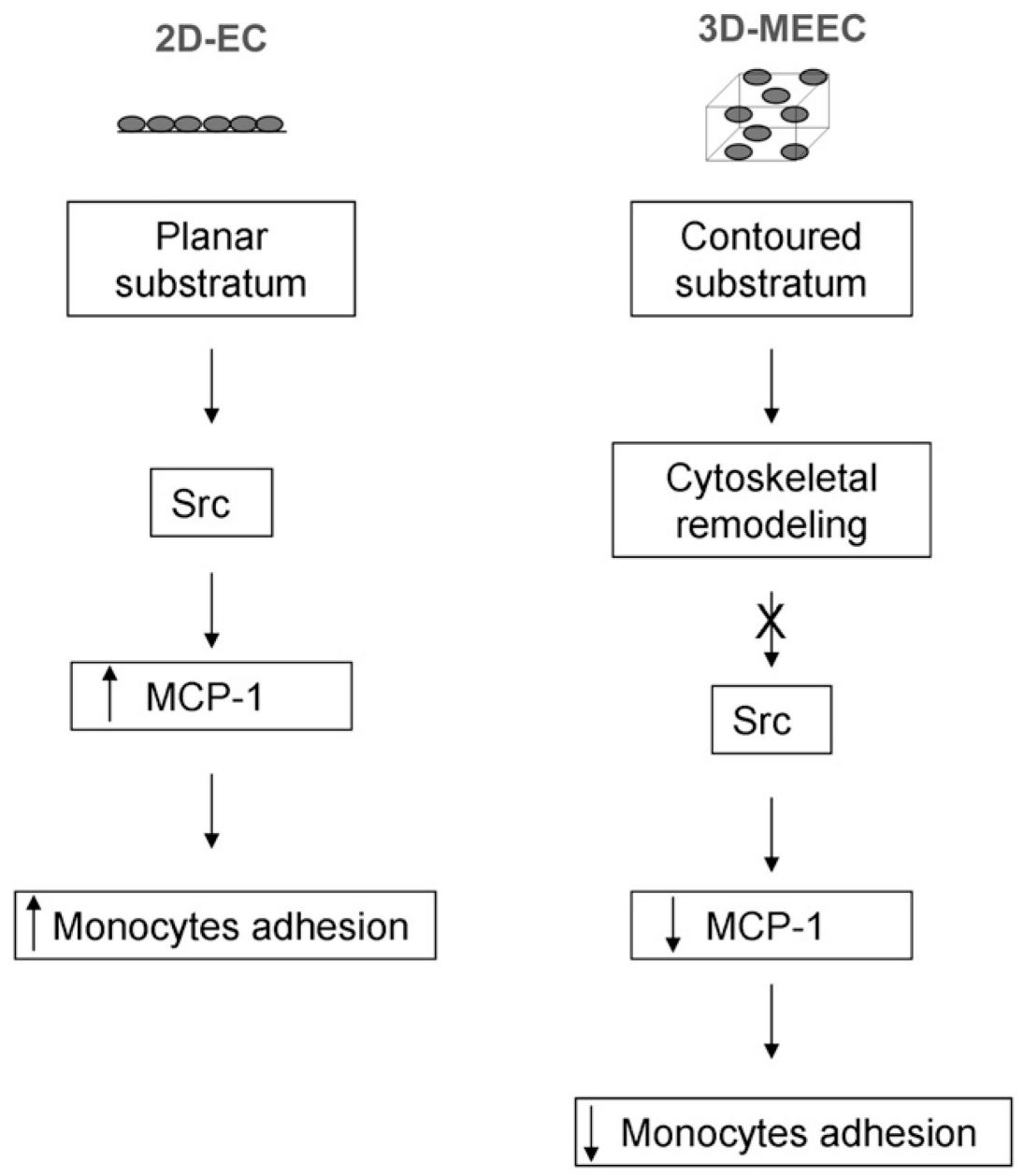

Fig. 7.

Scheme of the proposed pathway altered by the contoured subjacent surface sensed by the endothelial cells. The contoured topology of the substratum imposes a different cytoskeletal organization to ECs, which in turn interferes with the Src intracellular signaling. Reduction of secreted MCP-1 level, in turn, hinders monocytes adhesion to the site of inflammation. In contrast, in flat domain the Src signaling is not affected, therefore higher levels of MCP-1 and adherent monocytes are detected. 